\title{
Retracted: Analysis Model of Personality and Psychological Characteristics of Network Users under High-Pressure Working Environment
}

\author{
Security and Communication Networks
}

Received 16 November 2022; Accepted 16 November 2022; Published 30 November 2022

Copyright ( $\odot 2022$ Security and Communication Networks. This is an open access article distributed under the Creative Commons Attribution License, which permits unrestricted use, distribution, and reproduction in any medium, provided the original work is properly cited.

Security and Communication Networks has retracted the article titled "Analysis Model of Personality and Psychological Characteristics of Network Users under HighPressure Working Environment" [1] due to concerns that the peer review process has been compromised.

Following an investigation conducted by the Hindawi Research Integrity team [2], significant concerns were identified with the peer reviewers assigned to this article; the investigation has concluded that the peer review process was compromised. We therefore can no longer trust the peer review process, and the article is being retracted with the agreement of the Editorial Board.

\section{References}

[1] B. Yang, "Analysis Model of Personality and Psychological Characteristics of Network Users under High-Pressure Working Environment," Security and Communication Networks, vol. 2022, Article ID 2050181, 10 pages, 2022.

[2] L. Ferguson, "Advancing Research Integrity Collaboratively and with Vigour," 2022, https://www.hindawi.com/post/advancingresearch-integrity-collaboratively-and-vigour/. 


\title{
Analysis Model of Personality and Psychological Characteristics of Network Users under High-Pressure Working Environment
}

\author{
Bin Yang $\mathbb{D}^{1,2}$ \\ ${ }^{1}$ WenZheng College of Soochow University, Suzhou, China \\ ${ }^{2}$ School of Education, Soochow University, Suzhou, China \\ Correspondence should be addressed to Bin Yang; 2018210289@mail.chzu.edu.cn \\ Received 15 January 2022; Revised 26 January 2022; Accepted 4 February 2022; Published 7 March 2022 \\ Academic Editor: Muhammad Arif \\ Copyright (c) 2022 Bin Yang. This is an open access article distributed under the Creative Commons Attribution License, which \\ permits unrestricted use, distribution, and reproduction in any medium, provided the original work is properly cited. \\ Based on the prediction of social network psychological indicators, this paper studies the analysis model of network users' \\ personality and psychological characteristics under a high-pressure working environment. Through the analysis of five common \\ network users' personalities, the characteristics of personality psychology are extracted. Combined with the high-pressure \\ working environment, this paper analyzes the characteristics of network users' personalities and psychology, collects the \\ characteristics of network users' personality themes, and analyzes the characteristics of the dynamic extended dictionary. In order \\ to ensure the accuracy of feature analysis, the author's LDA algorithm is used to predict the network short text psychological index \\ of network users under a high-pressure working environment. The experimental results show that the model designed in this \\ paper is better than the traditional method in practical application.
}

\section{Introduction}

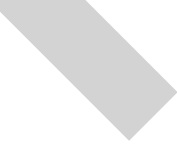

With the continuous development of the Internet, various network applications are becoming more and more popular. As a platform for online content publishing and dissemination, the network has brought new changes to people's social lives and communication methods. At the same time, the changes in human psychology and personality will be affected under the high-pressure working environment that will be displayed in the network social platform [1]. In order to better ensure national mental health, the design of the analysis model of the personality and psychological characteristics of the network users under the high-pressure working environment is proposed [2]. The traditional model adopts a questionnaire survey, which is not suitable for large-scale users to measure personality. Methods by acquiring the personalities of Internet users, we can further promote the development of related applications. Users may collect the psychological characteristics of network users more efficiently by using the network platform to automatically produce a large number of behavior data and forecast psychological characteristics based on network user behavior data [3]. This study studies the network behavior of microblog users, extracts user behavior features, creates a personality analysis model of microblog users, and confirms the model's viability through experiments, using the microblog as the platform for network research.

\section{Collection of Personality and Psychological Characteristics of Network Users under High- Pressure Working Environment}

By explaining the internal characteristics of external behavior, this paper studies human personality from the perspective of psychology. In order to better analyze the personality and psychological characteristics of network users under high-pressure working environment, the common personality categories are first analyzed and studied [4]. The key to combining the theory of psychological characteristics with social network analysis is to analyze and predict the users' psychological characteristics by analyzing the user data in the social network. From the perspective of personality research, personality psychology 
can be divided into different theoretical schools, including psychoanalysis school, trait school, biological school, behavior school, social learning school, humanism school, and cognitive school [5]. Trait school is one of them, and it gives a scientific quantitative standard for assessing personality. The school of psychological characteristics produces the most generally used psychological feature models in the study of social network users' psychological features, such as the psychological characteristics model and the big five psychological characteristics model [6]. According to the theory of types of psychological characteristics, people's behavior is divided into several fixed categories. That is to say, people who choose one kind of person are totally different from those who choose another [7]. Using the method of lexicology, this paper mainly uses the clustering algorithm in data mining to cluster the adjectives describing personality characteristics, forming five dimensions of personality traits. The big five personality model was used to measure the personality characteristics of microblog users, as shown in Table 1.

In order to study the personality and psychological characteristics of users in the network under high-pressure working environment, it is necessary to understand their psychological characteristics of network use [8]. In the process of personality psychological analysis, the measurement of psychological characteristics is the premise of psychological characteristic analysis. Generally, there are two ways to measure personality: self-active report and behavior passive analysis. Based on this, using the network behavior to predict personal personality, through interviews and questionnaires on personal personality data collection, and marking the data using the SCL-90 psychological well-being scale, positive emotion scale, life happiness scale, life satisfaction scale, mental health scale, stress ideation scale, etc. To ensure the accuracy of the research findings, the detailed information for each dimension is listed again, and a network personality psychological index dimension is created, as shown in Table 2, to accurately analyze the distribution of user personality psychological characteristics in a high-pressure working environment.

\section{Classification Algorithm of Network Personality Psychological Characteristics}

Taking microblogs as an example, this paper studies the five personality patterns of microblog users and collects sample data under the authorization of users. Then, the relationship between network personality and work stress is extracted by user relationship analysis [9]. The user's psychological characteristics are put into the dataset, the data are labeled according to the user's psychological characteristics, and the user's feature vector is obtained. The analysis process of network personality characteristics is shown in Figure 1.

Furthermore, the network user characteristics are extracted, analyzed, and input into the user personality analysis module. The output optimization model is trained according to the upper personality analysis model, and the analysis output results of five user personality tags are generated. The specific classification method is shown in Figure 2.

Furthermore, the data collected in the initial stages of login and construction is recorded, the Hadoop feature distribution of the collected information is carried out in the MySQL database, and the program calling database interface is written. In order to ensure the operational effect of the analysis model, the confusion value of network information is removed. We set $P$ as the amount of information sent by network users, the confusion value is $N_{d}$, and the interference index is $w_{d}$. In the process of psychological characteristic analysis and evaluation, it is necessary to meet the following requirements:

$$
\operatorname{perplexity}\left(D_{\text {test }}\right)=\exp \left\{\frac{-\sum_{d} \lg \left(P\left(w_{d}\right)\right)}{\sum_{d} N_{d}}\right\} \text {. }
$$

The Pearson correlation coefficient method is used to calculate the correlation coefficient between stress and psychological characteristics, and the symbol $R$ is used to represent the linear correlation between variables $X$ and $Y$. The Pearson correlation coefficient is defined as the quotient of covariance $\sigma_{X}$ and $\sigma_{Y}$, the standard deviation between two variables.

$$
\rho_{X, Y}=\frac{\operatorname{cov}(X, Y)}{\sigma_{X} \sigma_{Y}}=\frac{E\left[\left(X-\mu_{X}\right)\left(Y-\mu_{Y}\right)\right]}{\sigma_{X} \sigma_{Y}} .
$$

By estimating the covariance $X_{i}$ and standard deviation $Y_{i}$ between samples, the correlation coefficients are obtained as follows:

$$
r=\frac{\operatorname{perplexity}\left(D_{\text {test }}\right) \sum_{i=1}^{n}\left(X_{i}-\bar{X}\right)\left(Y_{i}-\bar{Y}\right)}{\rho_{X, Y} \sqrt{\sum_{i=1}^{n}\left(X_{i}-\bar{X}\right)^{2}} \sqrt{\sum_{i=1}^{n}\left(Y_{i}-\bar{Y}\right)^{2}}} .
$$

Based on the above algorithm, the linear correlation between work stress and personality psychological characteristics is further described. The correlation coefficient is calculated for any two $X, y$ variables in the sample data to get their corresponding variables.

If the correlation coefficient $r=0$, the relationship between $X$ and $Y$ does not hold.

When the correlation coefficient $r>0$, there is a positive correlation between two variables.

When the correlation coefficient $r<0$, the correlation between the two variables is negative.

Because the correlation coefficient has the property of the correlation coefficient method, it can be used to measure the linear correlation between variables [10]. Therefore, correlation coefficient analysis can be used to select features and find feature sets with strong linear correlations with the original data labels. The correlation coefficient approach, on the other hand, can only choose the best feature people, not the best overall categorization. The personality psychological characteristics are assessed according to the demands of the model application in order to verify the model's viability and use the work pressure and personality psychological characteristics index to evaluate it [11]. The accuracy of the model is an important standard to measure the effectiveness of the analysis method of users' psychological characteristics 
TABLE 1: Characteristics of big five personalities.

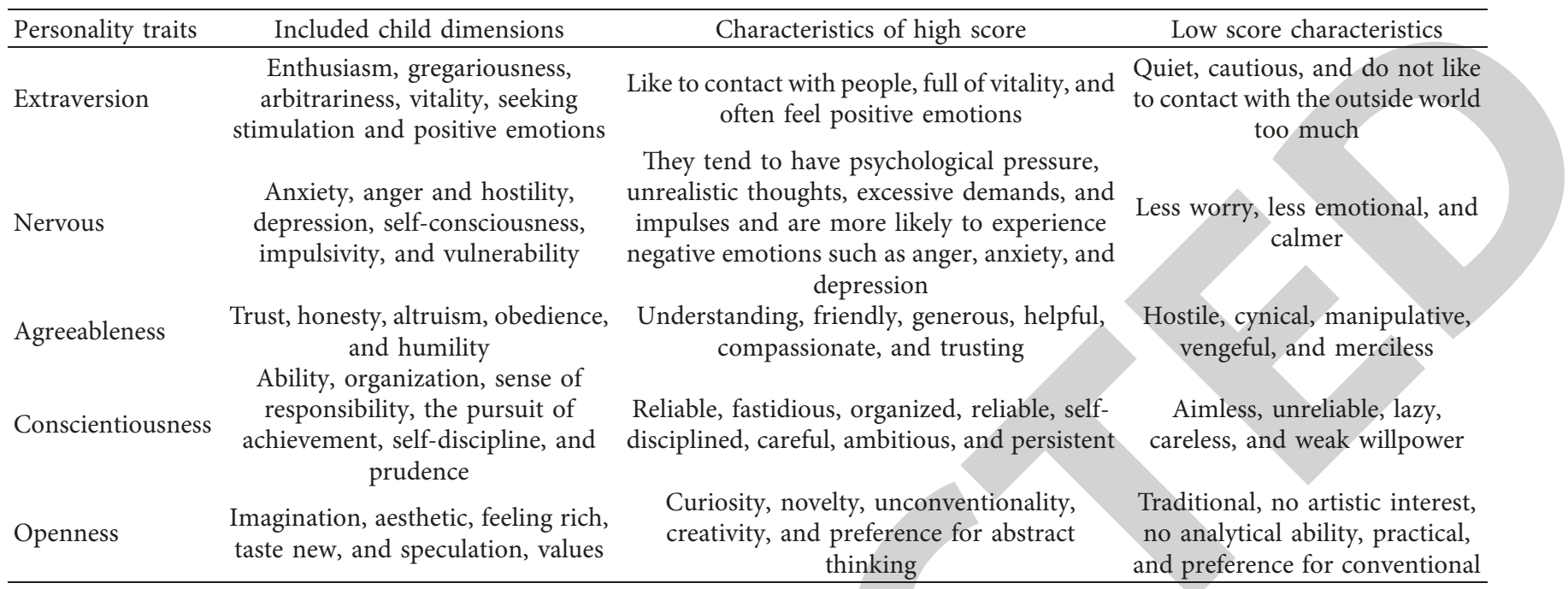

TABLE 2: Dimension information of network personality psychological indicators.

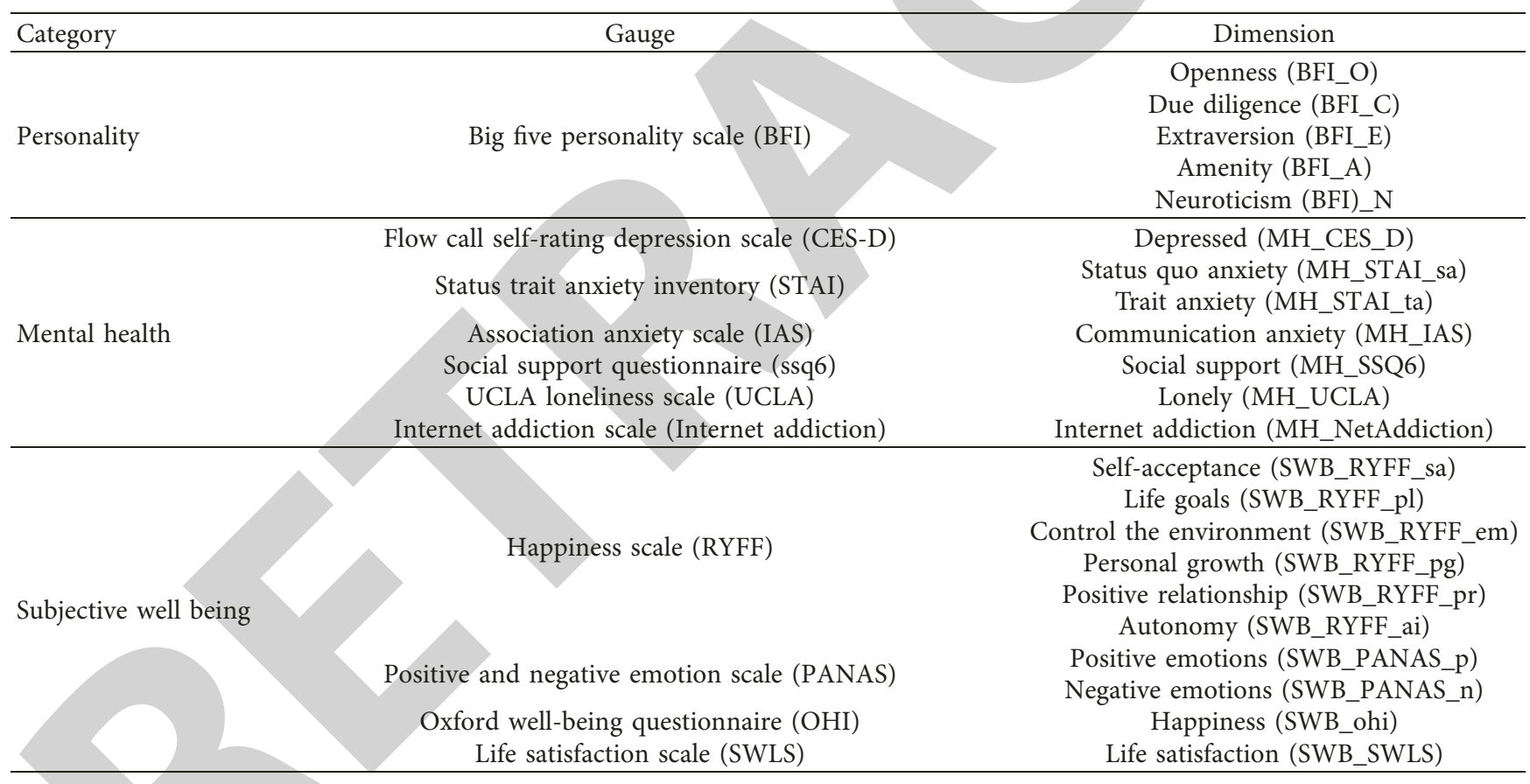

in the network during the analysis process, because the higher the accuracy of the model, the better it can describe the user's psychological characteristics when classifying them [12]. However, if the correctness of the model is taken as the only criterion to evaluate the merits and demerits of the network user personality analysis algorithm, a large number of false-negative data will be generated when the user personality analysis is carried out on an unbalanced dataset, which is inconsistent with the purpose of network user personality analysis. On this basis, several evaluation indexes of social network users' psychological characteristics analysis model are selected: model precision, model recall rate, and model $F 1$ value, and the evaluation formula of these three indicators is given.

$$
\begin{aligned}
\text { Precision } & =\frac{\text { True positive }}{\text { True positive }+ \text { False positive }}, \\
\text { Recall } & =\frac{\text { True positive }}{\text { True positive }+ \text { False negative }}, \\
F 1 & =\frac{2 \times \text { Precision } \times \text { Recall }}{\text { Precision }+ \text { Recall }} .
\end{aligned}
$$

Among them, true positive refers to the sample correctly identified as a positive class by the model, true positive refers to the sample identified as a negative class by model error, and false negative refers to the sample identified as a negative class by model error [13]. False-positive indicates the 


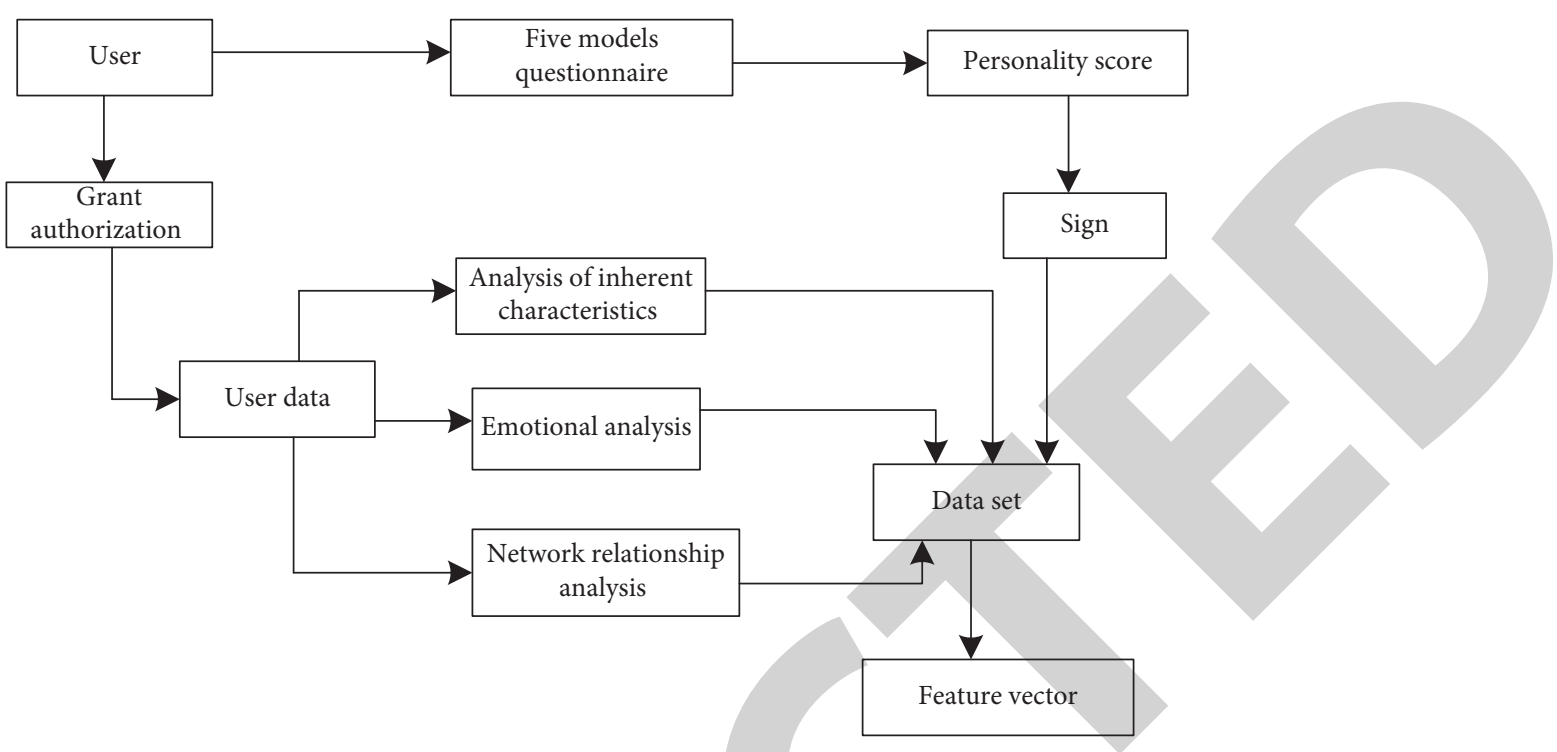

FIGURE 1: Network personality analysis process.

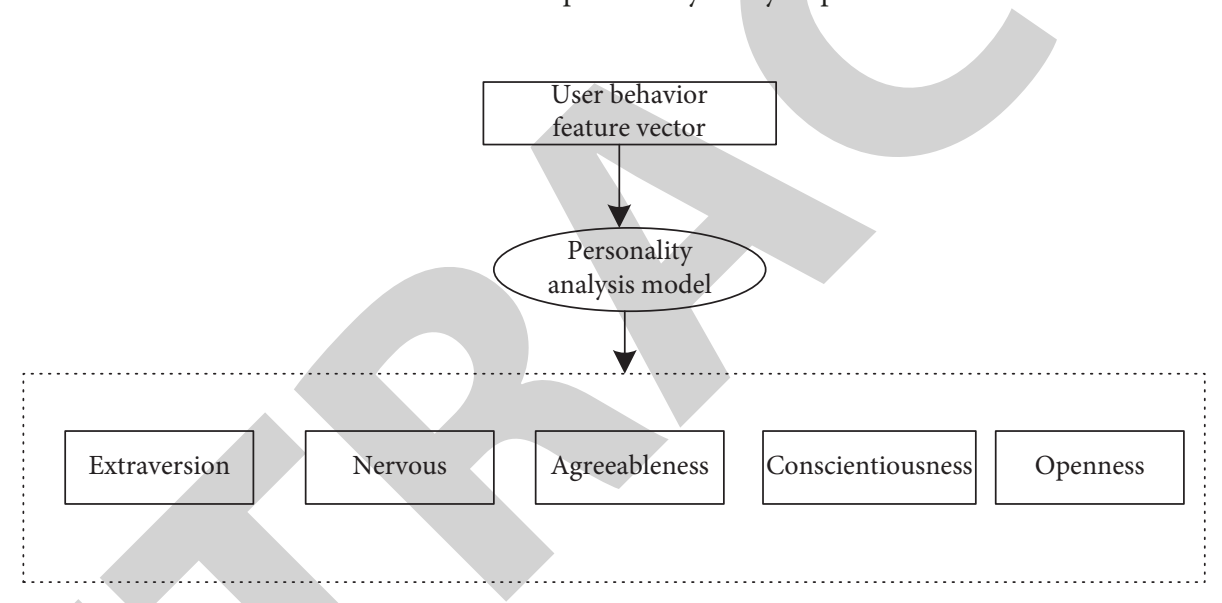

FIgURE 2: Classification of network personality characteristics.

proportion of positive classes correctly identified in all models; recall represents the proportion of positive classes correctly identified in all datasets; the value of the $F 1$ model is obtained by the weighted average of accuracy and recall rate [14]. Taking the accuracy rate, recall rate, and $F 1$ value as evaluation indexes can better reflect the performance of the analysis model of network users' psychological characteristics and provide a reference for the final selection of the model.

\section{Realization of the Analysis of the Personality and Psychological Characteristics of Network users}

Psychological characterization analytic procedures, such as data preprocessing, feature extraction, feature optimization, model construction, model training and cross-validation, and training model application, are used to analyze network users' personality and psychological traits [15]. In order to ensure the analysis effect of personality and psychological characteristics of network users, the changes in personality and psychological characteristics under high-pressure environments are analyzed first, as shown in Figure 3.

We preprocess the user's original network behavior data, extract the user's attributes, and get the associated feature vector. Furthermore, we improve the feature selection algorithm, create a binary classification model, train the classification model with the optimized feature vector, get the final classification model after 10 cross-validations, extract the user feature classification model as a test set, and finally use it at the heart of the physical and psychological features. They are classified, and psychological characteristics are analyzed. From a technical point of view, there needs to be a program that can extract data from the network on a large scale and store it in an appropriate way [16]. In social networks, from the perspective of modeling, heterogeneous data needs to be cleaned up and transformed into structured data. The data processing architecture is shown in Figure 4.

In order to ensure the high availability of data, the original user behavior dataset on the network needs to be cleared, invalid data needs to be filtered, and user data is deleted, including incomplete field information, insufficient number of blog posts, and so on. On the basis of data 


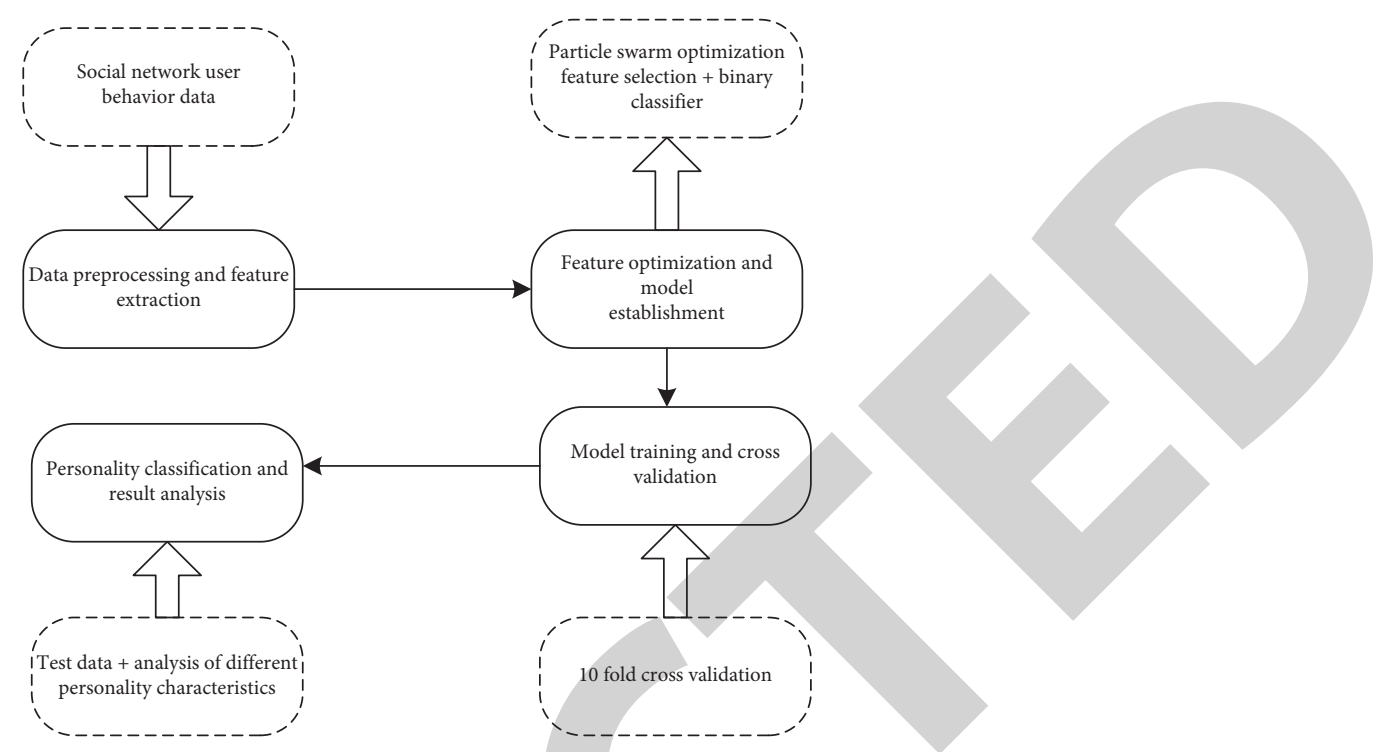

Figure 3: Changes in personality and psychological characteristics under high-pressure environments.

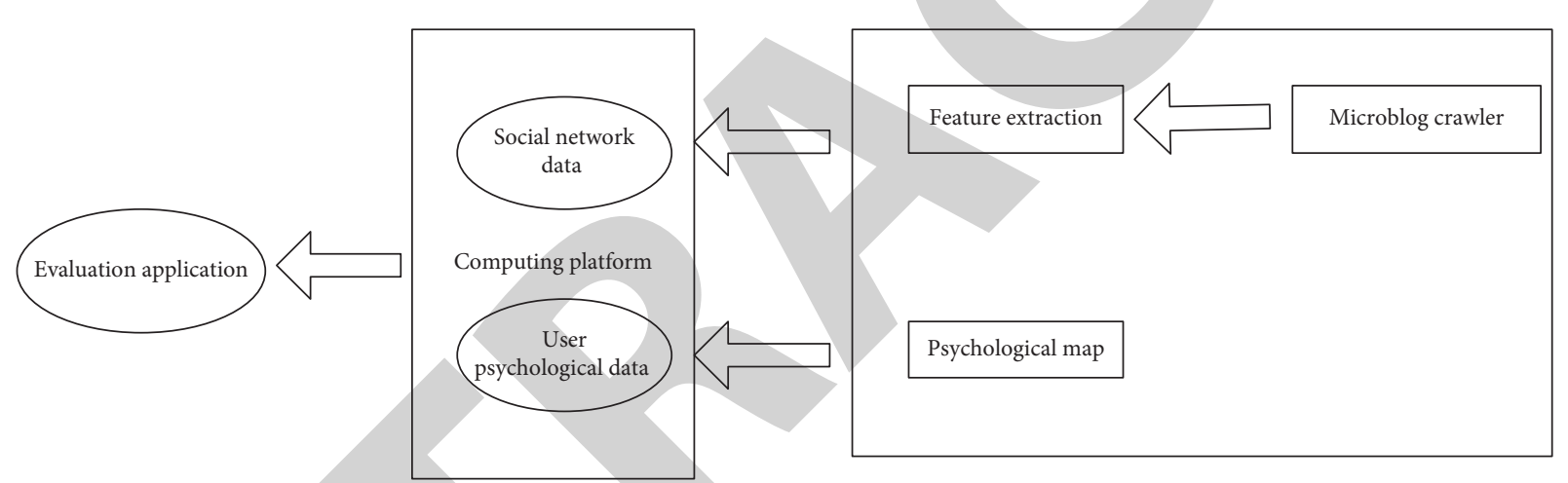

FIGURE 4: Data processing framework of personality and psychological characteristics of network users.

preprocessing, user behavior features are further extracted [17]. User behavior features can be divided into six types: time features, network features, part of speech tagging features, statistical features, discourse style features, and psychological discourse features. A personality analysis model is established based on the binary particle swarm optimization algorithm [18]. Thus, the functional framework structure of the network user psychological characteristics analysis model is designed in detail, as shown in Figure 5.

The offline dataset of known tags are stored in the offline database, and the feature extraction module is used to extract features: the obtained user feature vector is input into the training and testing module of the personality analysis model, and the obtained optimal personality analysis model is output to the network user personality analysis module; the obtained user data is input into the network user behavior data analysis module [19]. In the psychological characterization analysis system, network users will produce a lot of behavioral data when using the network, which provides the data basis for feature extraction. The backup user data acquisition and input module are mainly to realize the automatic collection of the original behavior data. Based on the analysis of the psychological characteristics of microblog social network users, this paper proposes the authorization requirements for microblog account users [20]. When the access is finished, the module will obtain the network data related to the user account, including user attributes and user behavior data, and output the obtained user data to the feature extraction module through the Selenium browser automatic test framework. Figure 6 shows the specific steps.

Based on the above steps, the text style features of the user network are marked, including exclamation marks, punctuation marks, part of speech marks, and vocabulary marks. The characteristics of tags are expressed according to the frequency of user articles. The mental lexicon features are extracted from the LIWC dictionary, and the TF-IDF value of the mental lexicon is calculated. In this paper, TF-IDF is used to extract mental lexicon features for the classification of personality psychological characteristics, as shown in Figure 7.

Furthermore, KNN, decision trees, and Naive Bayes are used to train the personality analysis model. After several models are trained, a set of performance test data is input. When the evaluation index reaches the expected goal, the final analysis model of psychological characteristics is 


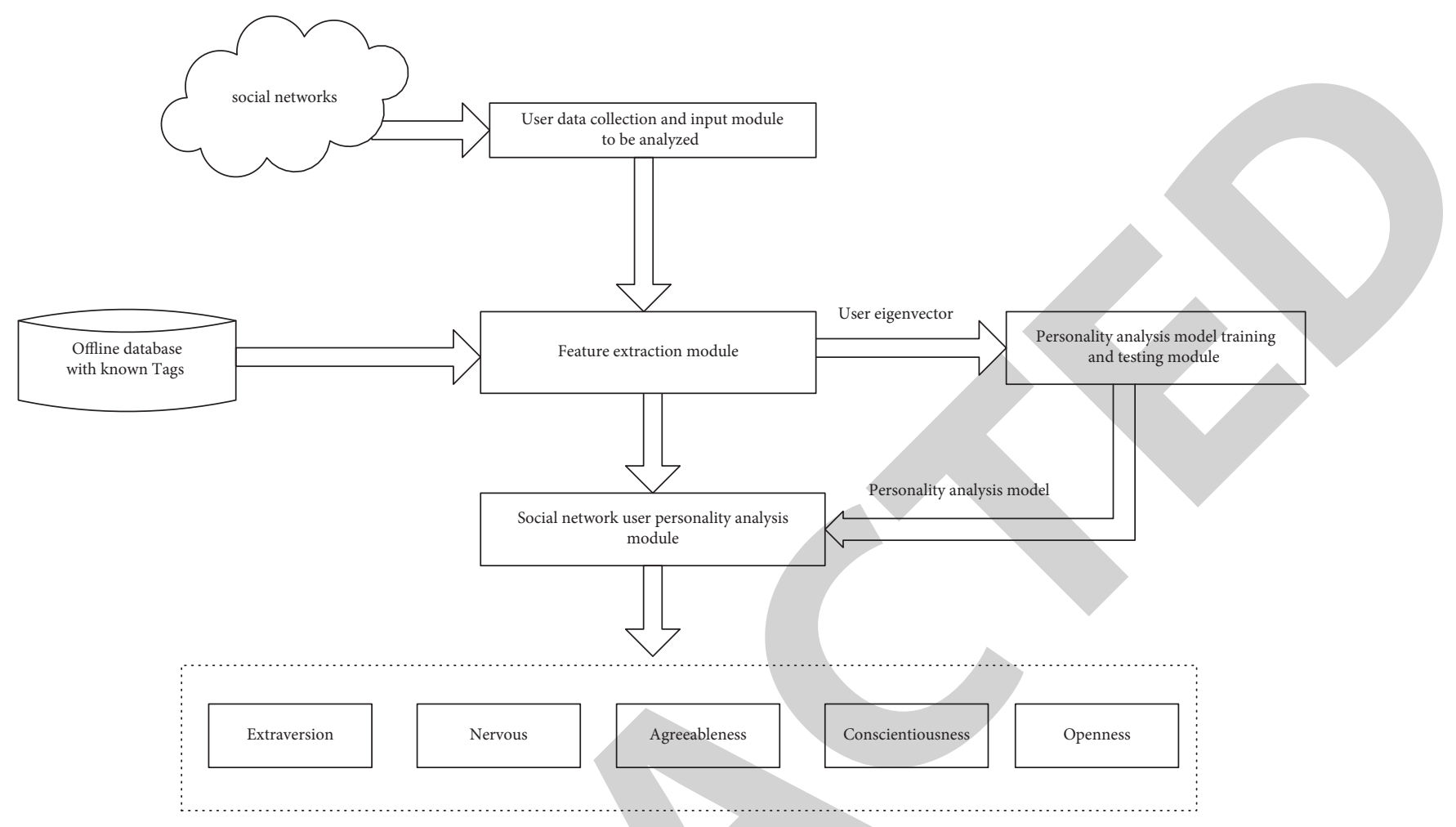

Figure 5: Functional framework of network users psychological characteristics analysis model.

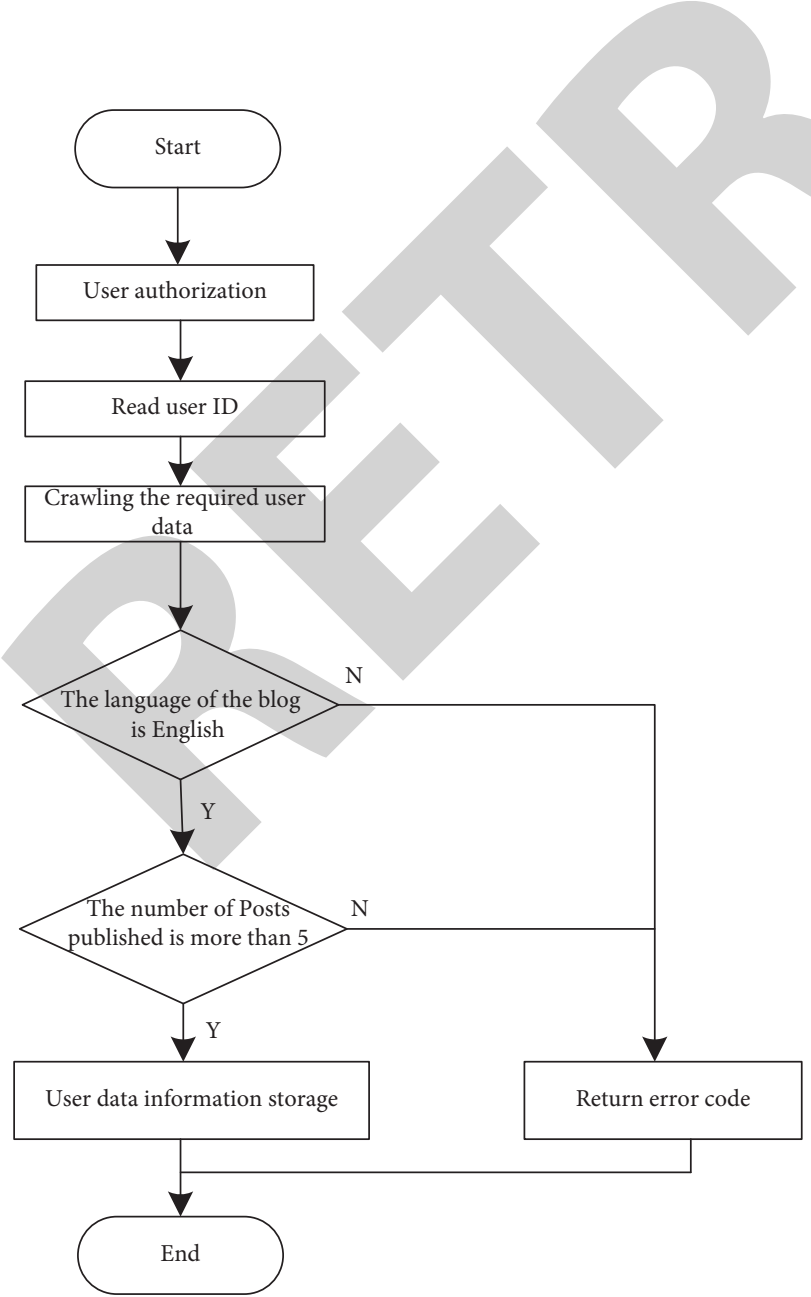

Figure 6: Network user characteristic data mark.

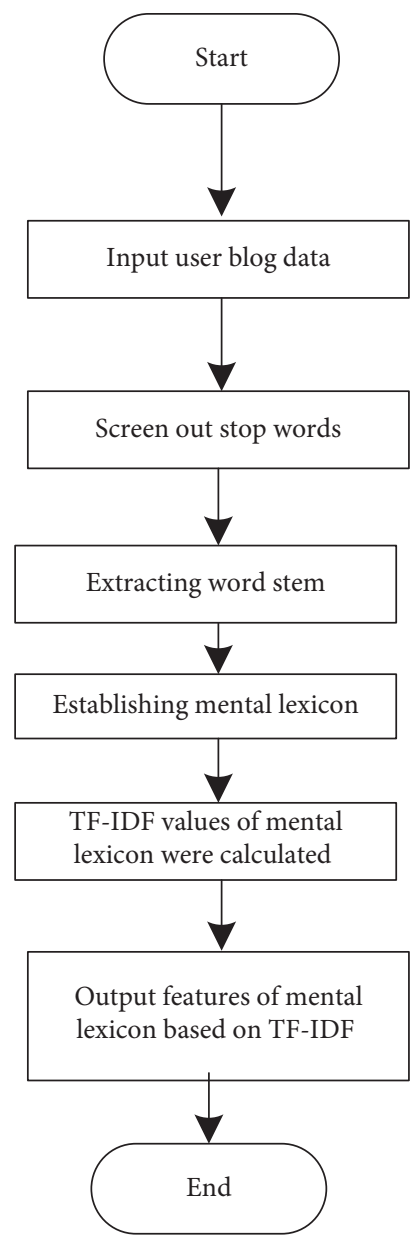

Figure 7: Analysis of network users' vocabulary characteristics. 
output, and the user's psychological characteristics in the analysis data are classified. If the expected goal is not achieved, the training and optimization of the psychological characteristics analysis model will be continued. At the same time, the training and testing module of the personality analysis model stores the best model in the history training model by default and outputs it to the character analysis module of network users. Based on this, the analysis steps of personality and psychological characteristics of network users are improved, as shown in Figure 8.

Social network user data characteristics will change with the development of technology. On this basis, a network user characteristic analysis system based on an offline database is designed to realize the continuous collection of new user data. Through continuous training and optimization of the analysis model of the psychological characteristics, the analysis model of training samples of the personality psychological characteristics analysis model is optimized to obtain better psychological characteristic analysis results.

\section{Analysis of Experimental Results}

It is necessary to analyze the psychological factors of users and to make a systematic analysis of users' psychological factors. For index data, there are many kinds of network data, including population information, text, pictures, network data, etc. What kind of data is chosen as the key point determines the computer fields involved: image content, computer graphics, network content, complex network, text content, etc., can be processed with natural language. Therefore, this step is very important for feature selection. Compared with microblog data, text data were chosen as the research focus. In the network, the text is the most common data source. Because microblogging can see text, it can avoid privacy issues. In addition, due to the external visibility of microblog text, the difficulty of crawling is the lowest. Based on this, the sample information is collected.

In order to ensure the effectiveness of the experimental research, we use an Intel i5-5200u processor and an 8 GB memory system to implement Python 2.7.9 on the Windows 10 operating system. This experiment uses a mypersonality dataset to verify the above algorithm. Mypersonality is a third-party application for microblogging. Through the authorization of users, they let users participate in psychological tests, get feedback on test results, and collect resume information on the microblog. Because some statistical features are extracted from the posts on microblogs, only a few blog user data can provide enough information for the model. Therefore, the user data of at least 5 blog posts are selected as experimental data. After screening, this set of data contains 9809 articles from 205 microblogs. The distribution of personality type is shown in Table 3. The brain's ext, neu, agr, con, and OPN represent five personality types: extrovert, nervous, friendly, responsible, and outgoing.

In order to verify the correctness of the analysis model in this paper, the LIWC feature and psy feature are used as the inputs of the model, and the precision, recall rate, and $F 1$ are taken as the evaluation indexes, and the accuracy, recall rate

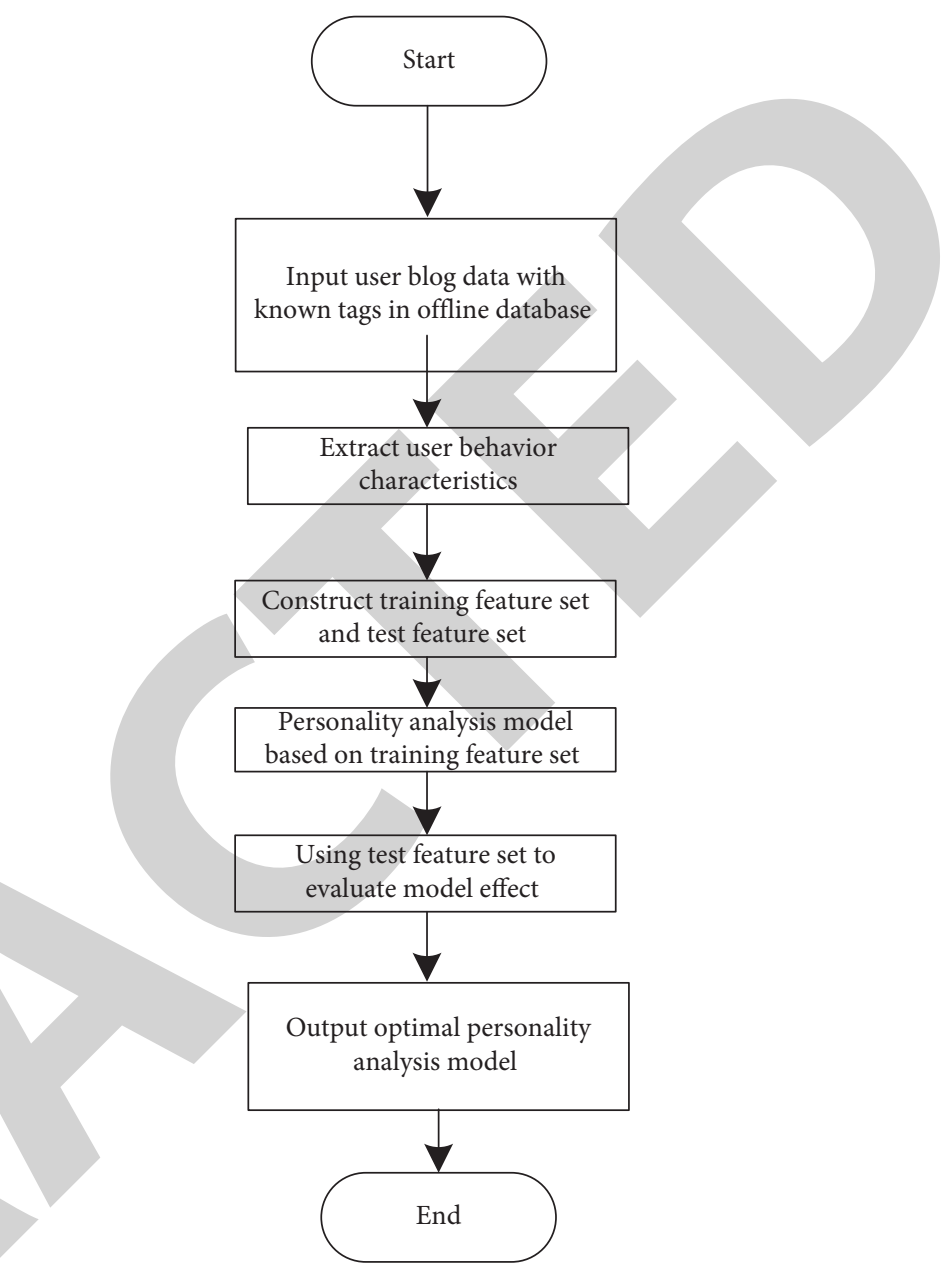

FIgURE 8: Training process of the personality and psychological characteristics analysis model.

TABLE 3: Distribution of personality characteristics of datasets.

\begin{tabular}{lccccc}
\hline Tag paper & Ext & Neu & Agr & Con & Opn \\
\hline 1 & 82 & 77 & 113 & 107 & 146 \\
0 & 123 & 128 & 92 & 98 & 59 \\
\hline
\end{tabular}

and other parameters of the model classification are tested and recorded, as shown in Table 4.

After unified processing based on the data in the above table, the analysis results of the traditional model and the model in this paper are recorded in $\mathrm{dB}$, and the details are shown in Table 5.

Analysis diagrams are drawn according to Tables 5 and 6, and conducted a more intuitive comparative analysis as shown in Figures 9-11.

According to Figures 9 to 11, it can be seen that the accuracy rate of the method in this paper is higher than that of the traditional method in the EXT psychological characteristics of the other feature, and its value is $0.26 \mathrm{~dB}$ higher. In other comparative analyses, it can be seen that the accuracy rate of the method in this paper is the features of Ext, $\mathrm{Neu}$, Agr, and Con are basically $0.1 \mathrm{~dB}$ higher than the traditional method, and some are even higher than $0.2 \mathrm{~dB}$. 
TABLE 4: Classification effect of personality and psychological characteristics of network users.

\begin{tabular}{lcccccc}
\hline Evaluating indicator & Feature set & Ext & Neu & Agr & Con & Opn \\
\hline \multirow{2}{*}{ Precision } & LIWC & 0.58 & 0.52 & 0.52 & 0.48 & 0.60 \\
& psy & 0.52 & 0.57 & 0.58 & 0.58 & 0.55 \\
\hline \multirow{2}{*}{ Recall } & LIWC & 0.53 & 0.55 & 0.53 & 0.47 & 0.62 \\
& psy & 0.53 & 0.60 & 0.58 & 0.57 & 0.58 \\
& LIWC & 0.53 & 0.51 & 0.48 & 0.44 & 0.60 \\
& psy & 0.51 & 0.57 & 0.57 & 0.54 & 0.56 \\
\hline
\end{tabular}

TABLE 5: Analysis accuracy of personality and psychological characteristics of network users in this model.

\begin{tabular}{lccccrr}
\hline Features & Model & Ext & Neu & Agr & Con & Opn \\
\hline \multirow{3}{*}{ Other } & KNN & 0.70 & 0.74 & 0.80 & 0.73 & 0.76 \\
& NB & 0.86 & 0.75 & 0.80 & 0.73 & 0.73 \\
\hline \multirow{3}{*}{ Other + psy } & DT & 0.73 & 0.70 & 0.74 & 0.59 & 0.71 \\
& KNN & 0.75 & 0.87 & 0.74 & 0.73 \\
\hline \multirow{3}{*}{ Other + style } & NB & 0.71 & 0.80 & 0.75 & 0.76 & 0.78 \\
& KN & NN & 0.70 & 0.78 & 0.79 & 0.73 \\
\hline
\end{tabular}

TAвLe 6: Analysis accuracy of personality and psychological characteristics of network users under the traditional model.

\begin{tabular}{|c|c|c|c|c|c|c|}
\hline Features & Model & Ext & Neu & Agr & Con & Opn \\
\hline \multirow{3}{*}{ Other } & KNN & 0.69 & 0.63 & 0.58 & 0.60 & 0.71 \\
\hline & NB & 0.60 & 0.62 & 0.55 & 0.54 & 0.71 \\
\hline & DT & 0.62 & 0.62 & 0.60 & 0.59 & 0.72 \\
\hline \multirow{3}{*}{ Other + psy } & KNN & 0.69 & 0.63 & 0.57 & 0.60 & 0.71 \\
\hline & NB & 0.60 & 0.62 & 0.56 & 0.55 & 0.71 \\
\hline & DT & 0.63 & 0.62 & 0.55 & 0.57 & 0.71 \\
\hline \multirow{3}{*}{ Other + style } & $\mathrm{KNN}$ & 0.69 & 0.63 & 0.58 & 0.60 & 0.71 \\
\hline & $\mathrm{NB}$ & 0.60 & 0.63 & 0.55 & 0.55 & 0.71 \\
\hline & DT & 0.65 & 0.62 & 0.57 & 0.61 & 0.72 \\
\hline
\end{tabular}

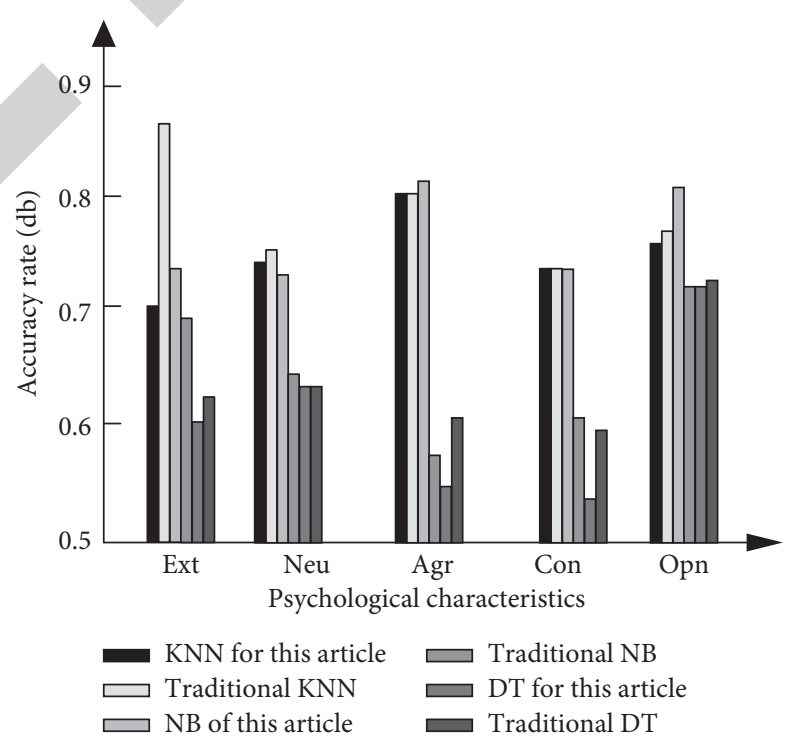

FIGURE 9: Accuracy rate of other characteristics analysis of user personality. 


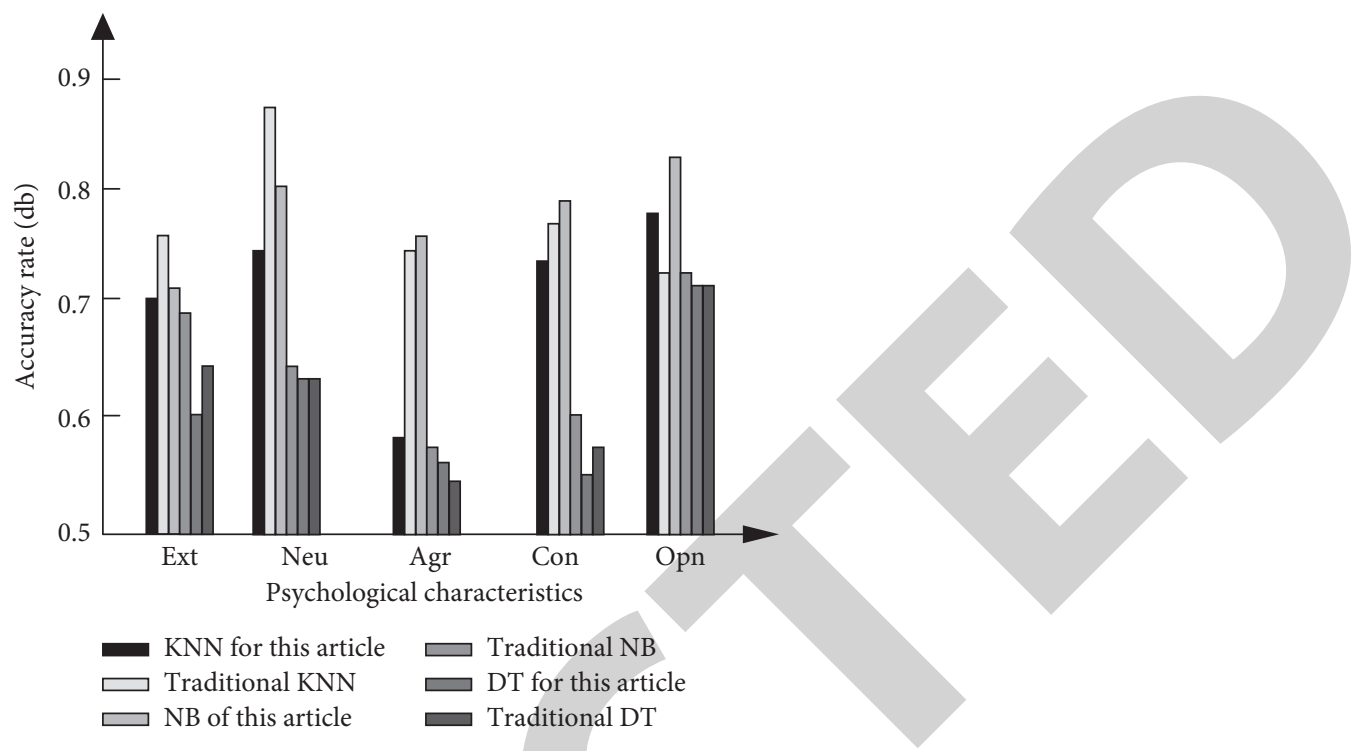

Figure 10: Accuracy rate of user character other + psy feature analysis.

The Opn characteristic is also higher than the traditional method, which is basically higher than $0.05 \mathrm{~dB}$. As a result, the procedure described in this article is in the real application process, and the analysis accuracy of the network user's personality and psychological features analysis model has been greatly enhanced. It can better analyze user changes. Simultaneously, it may assess the psychological aspects of a high-pressure work environment to assure users' psychological well-being that they are being timely and accurately monitored.

\section{Conclusion}

Taking the analysis of network users' psychological characteristics as the research content and taking microblog users as the research object, through the mining of network users' behavior characteristics, this paper proposes the design of a network users' personality and psychological characteristics analysis model under a high-pressure working environment. This study realizes the correlation analysis between the psychological characteristics of network users and the high-pressure working environment, and introduces the relationship between the psychological characteristics of the big five and psychological characteristics. This study discusses the research background and significance, describes the current psychological characteristics analysis technology based on network users, summarizes the research status of network users' psychological characteristics at home and abroad, summarizes the key features of network users' psychological characteristics analysis, reduces the workload of feature extraction, and improves the recognition efficiency and accuracy. 


\section{Data Availability}

The data used to support the findings of this study are included within the article.

\section{Conflicts of Interest}

The authors declare that they have no conflicts of interest.

\section{References}

[1] S. E. Williams, D. M. Sarno, J. E. Lewis, M. K. Shoss, M. B. Neider, and C. J. Bohil, "The psychological interaction of spam email features," Ergonomics, vol. 62, no. 8, pp. 983-994, 2019.

[2] B. Ahmad, M. Usama, C. M. Huang, K. Hwang, M. S. Hossain, and G. Muhammad, "Discriminative feature learning for skin disease classification using deep convolutional neural network," IEEE Access, vol. 8, p. 1, 2020.

[3] S. A. Mostafa, A. Mustapha, M. A. Mohammed, R. I. Hamed, N. Arunkumar, and Abd, "Examining multiple feature evaluation and classification methods for improving the diagnosis of Parkinson's disease," Cognitive Systems Research, vol. 54, pp. 90-99, 2019.

[4] S. Lou, Y. Feng, Z. Li, H. Zheng, and J. Tan, "An integrated decision-making method for product design scheme evaluation based on cloud model and EEG data," Advanced Engineering Informatics, vol. 43, Article ID 101028, 2020.

[5] D. Zhang, C. Wu, and J. Liu, "Ranking products with online reviews a novel method based on hesitant fuzzy set and sentiment word framework," Journal of the Operational Research Society, vol. 71, no. 3, pp. 528-542, 2020.

[6] P. Schmidt, A. Reiss, R. Dürichen, and K. V. Laerhoven, "Wearable-based affect recognition-a review," Sensors, vol. 19, no. 19, pp. 4079-4080, 2019.

[7] M. Hashemi and M. Hall, "Visualization feature selection machine learning identifying the responsible groupfor extreme acts of violence," IEEE Access, vol. 6, p. 1, 2018.

[8] L. He, D. Jiang, and H. Sahli, "Automatic depression analysis using dynamic facial appearance descriptor and d process Fisher encoding," IEEE Transactions on Multimedia, vol. 21, no. 6, pp. 1476-1486, 2019.

[9] S. Mohdiwale, M. Sahu, G. R. Sinha, and V. Bajaj, "Automated cognitive workload assessment using logical teaching learning-based optimization and PROMETHEE multi-criteria decision making approach," IEEE Sensors Journal, vol. 20, p. 1, 2020.

[10] A. Singh, S. U. Rehman, S. Yongchareon, and P. H. J. Chong, "Sensor technologies for fall detection systems a review," IEEE Sensors Journal, vol. 20, no. 13, pp. 6889-6919, 2020.

[11] G. Vila, C. Godin, O. Sakri et al., "Real-time monitoring of passenger's psychological stress," Future Internet, vol. 11, no. 5 , p. 102, 2019.

[12] A. Oker, N. Glas, F. Pecune, and C. Pelachaud, "An embodied virtual agent platform for emotional s effect experiments a proof of concept," Biologically Inspired Cognitive Architectures, vol. 24, no. 10, pp. 107-114, 2018.

[13] R. Munirathinam and S. C. Manoharan, "Analysis of brainstem in albl," International Journal of Biomedical Engineering and Technology, vol. 29, no. 1, p. 17, 2019.

[14] S. Shchebetenko, "Do personality characteristics explain the associations between self-esteem and online social networking behaviour?" Computers in Human Behavior, vol. 91, pp. 1723, 2019.
[15] S. M. Hegner, A. D. Beldad, and G. J. Brunswick, "Automatic we trust investigating the impact of trust control personality characteristics and extrinsic and intrinsic motivations on the acceptance of autonomous vehicles," International Journal of Human-Computer Interaction, vol. 35, no. 4, pp. 1-12, 2019.

[16] P. Adamopoulos, A. Ghose, and V. Todri, "The impact of user personality traits on word of mouth text-mining social media platforms," Information Systems Research, vol. 29, no. 3, pp. 612-640, 2018.

[17] P. Altanopoulou and N. Tselios, "Big five personality traits and academic learning in wiki-mediated collaborative activities," International Journal of Distance Education Technologies, vol. 16, no. 3, pp. 81-92, 2018.

[18] J. Sok, H. Hogeveen, A. R. W. Elbers, and A. G. J. M. OudeLansink, "Perceived risk and personality traits explaining heterogeneity in Dutch dairy farmers beliefs about vaccination against Bluetongue," Journal of Risk Research, vol. 21, no. 5-6, pp. 562-578, 2018.

[19] J. Trigg, K. Thompson, B. Smith, and P. Bennett, “Archetyping relationships with companion animals to understand disaster risk-taking propensity," Journal of Risk Research, vol. 22, no. 3-4, pp. 475-496, 2019.

[20] D. Toker, C. Conati, and G. Carenini, "Gaze analysis of user characteristics in magazine style narrative visualizations," User Modeling and User-Adapted Interaction, vol. 29, no. 6, pp. 1-35, 2019. 